\title{
El efecto del gobierno corporativo y la propiedad sobre el grado de orientación al mercado: evidencia empírica en el caso de Chile
}

The Effect of Corporative Governance and Property on the Degrees of Market Orientation: Empiric Evidence in the Chilean Case

O efeito do governo corporativo e a propriedade sobre o grau de orientação ao mercado: evidência empírica no caso do Chile

\author{
Ismael Oliva* \\ Karen Alarcón
}

Fecha de recibido: 24 de febrero de 2017

Fecha de aprobado: 15 de noviembre de 2017

Doi: http://dx.doi.org/10.12804/revistas.urosario.edu.co/empresa/a.5436

Para citar: Oliva, I., \&, Alarcón, K. (2018). El efecto del gobierno corporativo y la propiedad sobre el grado de orientación al mercado: evidencia empírica caso Chile. Universidad \& Empresa, 20(35), 79- 115.

Doi: http://dx.doi.org/10.12804/revistas.urosario.edu.co/empresa/a.5436

\section{RESUMEN}

Este artículo investiga si el gobierno corporativo y la propiedad actúan como determinantes del grado de orientación al mercado en un grupo de empresas chilenas. La muestra incluyó datos de 101 empresas registradas en la Bolsa de Comercio de Santiago en 2016, las cuales

* Director de la Escuela de Postgrado, Facultad de Economía y Negocios, Universidad de Chile. Profesor Asociado Docente. PhD en Business Administration, Aston Business School. Master en Business Administration, Massachusetts Institute of Technology (MIT). Ingeniero Comercial, Universidad de Chile, Santiago, Chile. Correo electrónico: ioliva@fen.uchile.cl

** Consultor e Investigador, Escuela de Postgrado, Facultad de Economía y Negocios, Universidad de Chile. Magíster en Marketing, Universidad de Chile. Profesional en Finanzas y Comercio Internacional, Universidad de la Sallé, Colombia. Correo electrónico: kalarcong@fen.uchile.cl 
fueron procesadas por análisis de correlación y regresión múltiple. Los resultados empíricos mostraron que los coeficientes de las variables de concentración y presencia de directores independientes no son significativos; el coeficiente relativo al tamaño de la junta demostró desempeñar un papel importante sobre el grado de orientación al mercado y sobre dos de sus dimensiones internas (orientación al cliente y coordinación interfuncional), lo que sugiere que el efecto marginal del grado de orientación al mercado es mayor cuando el número de directores en el tablero aumenta. Este estudio verifica el papel estratégico de la junta directiva, poniendo en relieve el impacto que este órgano de gestión tiene sobre el desempeño empresarial. Los resultados de esta investigación pretenden servir de base para futuros estudios que den importancia a los aspectos empresariales y organizacionales en la implementación de actividades orientadas al marketing.

Palabras clave: Orientación al mercado, gobierno corporativo, junta directiva, estructura de la propiedad.

\section{ABSTRACT}

This paper aims to determine if corporative governance and property act as determinant factors in the degrees of market orientation in a group of companies in Chile. The sample included data of 101 Chilean companies registered in the Santiago Stock Exchange in 2016. This data was processed by correlation analysis and multiple regression. The empiric results showed that the coefficients of the concentration and presence of independent chief executives variables are not meaningful. The relative coefficient to the size of the board showed that it plays an important role in the degrees of market orientation and on its two internal dimensions (degrees of market orientation and interfunctional coordination). This suggest that the marginal effect of the degrees of market orientation is bigger when the number of chief executives increases. This study verifies the strategic role of the board, highlighting the impact this management organ has on the performance of the company. The results of this research pretend to be a base for future studies that focus on the business and organizational aspects of implemented activities towards marketing.

Keywords: Market orientation, corporative governance, directive board, property structure.

\section{RESUMO}

Este artigo investiga se o governo corporativo e a propriedade, atuam como determinantes do grau de orientação ao mercado em um grupo de empesas no Chile. A amostra incluiu dados de 101 empresas chilenas registradas na Bolsa de Comércio de Santiago em 2016, as quais foram processadas por análise de correlação e regressão múltipla. Os resultados empíricos mostraram que os coeficientes das variáveis de concentração e presença de diretores independentes não são significativos; o coeficiente relativo ao tamanho da junta demostrou desempenhar um papel importante sobre o grau de orientação ao mercado e sobre duas de suas dimensões interna (orientação ao cliente e coordenação interfuncional), o que sugere que o efeito marginal do grau de orientação ao mercado é maior quando o número de diretores no tabuleiro aumenta. Este estudo verifica o papel estratégico da junta 
diretiva, pondo em relevo o impacto que este órgão de gestão tem sobre o desempenho empresarial. Os resultados desta pesquisa pretendem servir de base para futuros estudos que deem importância aos aspetos empresariais e organizacionais na implementação de atividades orientadas ao marketing.

Palavras-chave: Orientação ao mercado, governo corporativo, junta diretiva, estrutura da propriedade.

\section{INTRODUCCIÓN}

La orientación al mercado ha recibido considerable atención en el mundo de académicos y profesionales; esto queda demostrado en el marco investigativo existente que soporta la importancia de esta variable. Desde principios de 1990 se han realizado avances en su conceptualización y medición; ejemplo de ellos son los aportes realizados por Bernard, Jaworski y Kohli (1993, y por Narver y Slater (1990), los cuales han sido marco de referencia para múltiples estudios enfocados en el impacto que tiene la orientación al mercado en el rendimiento de la empresa, y su importancia como vía para el éxito competitivo, (Dawes, 2000; McNaughton \& Osborne, 2001; Rapp, Schillewaert \& Hao, 2008). Por el contrario, a pesar de la importancia que tiene el conocer los determinantes del desarrollo de la orientación al mercado, es evidente la escasez de investigación enfocada en este campo en países emergentes. En el contexto chileno se han generado pocos aportes en temas relacionados con la gestión empresarial desde el punto de vista del marketing.

La creciente evidencia a nivel mundial de los efectos positivos de la orientación al mercado incrementa el interés por comprender los determinantes de la misma; es ahí donde la literatura expone cómo los factores internos son considerados de suma importancia, a razón de que el control sobre los aspectos que pueden influir en la orientación al mercado son manejados principalmente por gerentes o directivos (Kohli \& Jaworski, 1990; Santos, Vasquez, \& Alvarez, 2002). La estructura organizacional interna es considerada como uno de los recursos complementarios más importantes para el éxito de una estrategia organizacional; de esta forma, la evidencia literaria muestra el papel moderador que tienen las estructuras de propiedad en el desempeño de la orientación al mercado. En su estudio, Ho y Wu (2010) resaltan el importante papel de la gestión empresarial en el apoyo a la orientación al mercado de las compañías, pues esta actúa como un recurso, mientras el 
gobierno corporativo lo hace como una capacidad al interior de la compañía. Es la combinación de los recursos y capacidades lo que permite que las empresas logren resultados positivos. En base a lo anterior este estudio pretende evaluar de manera empírica si el gobierno corporativo que considera la junta directiva enfocada en la presencia de consejeros independientes y el tamaño de la misma, como la concentración de la propiedad (Ducassy \& Montandrau, 2015; Lemmon \& Lins, 2003; Mollah, Al Farooque, \& Karim, 2012; Shapiro \& Tang, 2015a), se presentan como determinantes en el grado de orientación al mercado y sobre sus dimensiones internas (orientación al cliente, orientación a la competencia y coordinación interfuncional), dando énfasis en la importancia que tienen los factores organizativos en las compañías y como estos pueden ejercer una gran influencia sobre el éxito de la Orientación al mercado.

Para ello, el estudio se centra en una muestra compuesta por 358 empresas que transan en la bolsa de valores de Santiago. Los datos de la variable dependiente orientación al mercado se recopilaron a través de la escala de tres dimensiones validada por Olavarrieta et al., (1999) en su estudio realizado en Chile, que sigue la conceptualización propuesta por
Narver y Slater (1190); el cuestionario fue administrado a dueños, socios o ejecutivos principales en cargos en las empresas seleccionadas. Posteriormente, se realizó un proceso de validación de la escala, un análisis de correlaciones y se desarrolló un modelo de regresión para el testeo de las hipótesis, con el fin de terminar con la discusión de las principales conclusiones y limitaciones. Esta investigación intenta contribuir a la literatura sobre orientación al mercado que como fue comentado es escasa en el entorno chileno. En primera instancia se consideran diferentes dimensiones de gobierno corporativo, proporcionando nuevas perspectivas e implicancias a tener en cuenta en la práctica empresarial. La literatura actual sobre estos temas aborda la realidad de América del Norte y otras economías occidentales, donde la mayoría de las empresas son privadas; sin embargo, en el caso de las economías emergentes, los aspectos de gobierno corporativo difieren, lo que hace que sus hallazgos no puedan generalizarse a las empresas en las economías menos desarrolladas, (Song, Wei, \& Wang, 2015a). Por lo tanto, da respuesta al siguiente cuestionamiento puede ayudar a las empresas a determinar la medida en que aspectos específicos de su gobierno corporativo influyen en la orientación al mercado, considerando 
el papel del gobierno corporativo en el ámbito de la administración de negocios y del marketing.

\section{MARCO TEÓRICO E HIPÓTESIS}

\subsection{Determinantes "Orientación al mercado"}

De acuerdo con Narver \& Slater (1990), la orientación al mercado reúne un sistema de valores seguidos por la organización, que se divide en tres categorías: orientación a los competidores, orientación a los consumidores y coordinación interfuncional. Esta investigación adopta las tres construcciones introducidas por Narver y Slater (1990) para medir la orientación al mercado de una empresa. La orientación al mercado está relacionada a los procesos específicos y rutinarios que crean valor superior a los clientes y permite a las empresas obtener una ventaja competitiva sostenible (Long, 2015).

A partir del estudio bibliográfico, la investigación chilena se ha centrado en tres aspectos, primero en la evaluación del grado de orientación al mercado en diferentes sectores; segundo, en el análisis de las consecuencias de la orientación al mercado en las empresas, y finalmente, en la construcción de modelos de eva- luación que permitan analizar la influencia de la orientación al mercado en el desempeño organizacional. (Olavarrieta et al., 1999; Valenzuela \& Villegas, 2013). La creciente evidencia de la existencia de una relación positiva en el desempeño de la compañía y la orientación al mercado genera un creciente interés por comprender los principales determinantes relacionados con la orientación al mercado, evidencia que se expone desde tiempo pasado hasta el presente en los resultados de investigaciones a nivel mundial (Bernard et al., 1993; Kanagasabai, 2008; Riliang Qu \& Ennew, 2005). Los determinantes de la orientación del mercado han sido un trabajo de gran importancia en la literatura. Kohli \& Jaworski, (1990) definen la orientación al mercado como la reunión de la organización en torno a la inteligencia de mercados como estrategia fundamental para el reconocimiento de las necesidades actuales y futuras del cliente, además de la difusión de dicha información por las demás áreas de la empresa.

La existencia de estudios que centran su atención en la influencia de factores identificados por Kohli y Jaworski (1990) es variada. Qu y Ennew (2005), hacen una evaluación de los determinantes de la orientación al mercado en el sector turismo en 
China, donde evalúan los antecedentes reconocidos en la obra de Kohli y Jaworski (1990) y realizan una propuesta que reúne diferentes factores (regulación gubernamental y la estructura de la propiedad). En sus resultados muestran que los factores relacionados con la regulación y los efectos que generan los acuerdos de gobierno corporativo (a través de la estructura de propiedad) tienen una relación positiva con la orientación al mercado.

Estudios aún más recientes evalúan la influencia de las juntas directivas sobre la orientación al mercado. En sus hallazgos, Indu \& Vishag, (2016) demuestran cómo el consejo de directores ejerce una influencia positiva sobre el grado de orientación al mercado. A pesar de la investigación colectiva que se ha dado a nivel mundial, la atención que se ha presentado en países latinoamericanos a dichos aspectos es limitada. Puntualmente, en Chile los estudios identificados en la búsqueda bibliográfica mencionados previamente no concentran su atención en el estudio de los determinantes de la orientación al mercado en el sector empresarial. Por lo tanto, en la siguiente sección se plantea cómo los componentes del gobierno corporativo pueden influir en el grado de orientación al mercado de la compañía.

\subsection{Gobierno corporativo como} determinante de la orientación al mercado

El gobierno corporativo es entendido como el enlace entre las normas y prácticas que normalizan el poder de los directores (Charreaux, 2006). La legitimidad de dicho poder engloba la eficiencia de los mecanismos de control externo, el mercado financiero, laboral, político y legal, así como los asuntos internos que están relacionados con el control de los accionistas, de los directores y de la junta directiva (Ducassya, 2014). Diferentes asociaciones alrededor del mundo indican que las estructuras efectivas de gobierno corporativo motivan a las empresas a crear valor por medio de la innovación y el desarrollo, garantizando una correcta rendición de cuentas y mejorando el desempeño y el cumplimiento de objetivos establecidos (Securities Market Association, 2010). Por su parte, múltiples investigaciones centradas en el gobierno corporativo indican la existencia de mecanismos internos de gobierno corporativo y su efecto sobre diferentes variables empresariales, la estructura de la propiedad y el consejo de directores o junta directiva (Ducassy \& Montandrau, 2015; Lemmon \& Lins, 2003; Mollah et al., 2012; Shapiro \& Tang, 2015a; Lefort \& Urzúa, 2008). De 
esta forma, el estudio contemplara dichos mecanismo en el objetivo de evaluar la implicancia del gobierno corporativo como determinante en el grado de orientación al mercado.

\section{a) Estructura de propiedad}

La estructura de la propiedad contempla en qué medida se distribuye la posesión de acciones entre los accionistas mayoritarios y los demás participantes de la empresa y estima la relación entre los principales propietarios y los dueños menores (De Miguel, Pindado, \& De La Torre, 2004). Múltiples trabajos empíricos subrayan el impacto del buen gobierno corporativo en la actual era de la globalización, donde la competencia envuelta en el mundo de los negocios hace que sea necesaria una gestión organizacional, con el fin de responder a los cambios del entorno y poder mantener la competitividad (Sanusi \& Pel, 2015). Según la hipótesis de "vigilancia activa", la concentración de accionistas reduce el oportunismo gerencial, lo que resulta en menos conflictos entre los directores y los accionistas (Friend \& Lang, 1988). En sus investigaciones sobre los efectos de la orientación del mercado y el buen gobierno corporativo en el desempeño gerencial, Sanusi \& Pel, (2015) indican que la estructura de propiedad es un importante determinante de los resultados empresariales, y concluyen que la estructura de la propiedad sirve como mecanismo interno para que las empresas puedan mantener recursos clave, orientados a las actividades que promulguen la orientación hacia el cliente. En tal caso, la concentración de la propiedad adquiere una importancia mayor en la salvaguarda de los intereses de los accionistas minoritarios (Hill \& Snell, 1989). Otros ejemplos de estudios resientes validan la importancia de la estructura de propiedad en el entorno corporativo y su influencia sobre el rendimiento de las compañías. Li, Chau y Lai, (2010), examina en su investigación como factores estratégicos (orientación al mercado y estructura de propiedad), pueden afectar la asimilación de la compañía a procesos de comercio electrónico. En sus resultados se ratifica la influencia moderadora del accionista mayoritario entre la orientación al mercado y la asimilación de comercio electrónico.

Wang y Shailer, (2015) plantean un marco teórico donde se sustenta cómo la acumulación de accionistas mayoritarios logra potenciar el interés de los mismos por controlar la malversación de la gestión, (Shleifer \& Vishny, 1997). Otros autores sugieren que el aumento de la concentración de la propiedad motiva a los 
accionistas mayoritarios a presionar a la administración, con el fin de que la compañía mejore y responda a las fuerzas del mercado, lo cual resultaría en una mayor orientación al mercado (Ralston, TerpstraTong, Terpstra, Wang \& Egri, 2006; Song et al., 2015); además, evalúan el efecto de la concentración de la propiedad de los accionistas en empresas públicas del estado sobre el grado de orientación al mercado, confirmando que la propiedad concentrada permite aumentar la capacidad de la empresa por realizar una gestión más responsable, lo que lleva a la empresa a responder a las fuerzas del mercado. Por su parte, Song, Wei y Wang (2015) aseguran que diferentes tipos de estructura de propiedad pueden fomentar las acciones innovadoras resultantes de una fuerte orientación al mercado. $\mathrm{Su}$ hipótesis principal se concentra en corroborar de qué manera un alto nivel de concentración puede motivar al propietario mayoritario a seguir de cerca la toma de decisiones de los gerentes y lograr amortiguar la predisposición de la gestión por convertir en estrategias de reducción de riesgos que pueden minimizar el interés de los accionistas potenciales (Bethel \& Liebeskind, 1993).

Liu, Li y Xue, (2011) estudian de qué manera la estructura de propiedad afecta la orientación estratégica de la compañía en su interés por facilitar el éxito de sus negocios internacionales. Sus resultados indican que la concentración de la propiedad es un predictor significativo de la orientación al mercado, y resultados resaltan cómo la alta concentración de la propiedad es un factor de desventaja, que impide el desarrollo de las actividades empresariales en las empresas de los mercados emergentes. Cuando la concentración de la propiedad es baja, los propietarios no cuentan ni con información necesaria, ni con el poder para monitorear las decisiones gerenciales con eficiencia (Hill \& Snell, 1989). Partiendo del estudio literario, es posible indicar que los resultados en mercados emergentes confirman una relación positiva entre la concentración de la propiedad y el grado de orientación al mercado, no siendo ajenos de los hallazgos contradictorios. El interés es confirmar o negar dicha relación con el objetivo de general resultados frente a la situación chilena. A partir del estudio literario realizado, proponemos la siguiente hipótesis:

\section{H1. La concentración de propie- dad se relaciona positivamente con el grado de orientación al mercado de la compañía.}

De igual forma, se pretende verificar la posible influencia de la variable 
sobre las dimensiones internas de la orientación al mercado, por lo que se proponen las siguientes hipótesis complementarias.

H1a. La concentración de propiedad se relaciona positivamente con el grado de orientación al cliente.

H1b. La concentración de propiedad se relaciona positivamente con el grado de orientación a la competencia.

H1c. La concentración de propiedad se relaciona positivamente con el grado de coordinación interfuncional.

\section{b) Estructura de la Junta directiva (Presencia consejeros independiente)}

El directorio es considerado la principal instancia del gobierno de la empresa, ya que no solo ejerce la administración de la compañía, representando los intereses de los accionistas, sino que sus decisiones juegan un papel importante en la creación de valor (Lefort, 2008). Por su parte, la junta ejerce control sobre los altos ejecutivos y maneja el tema de incentivos, mientras el consejo se encarga de examinar las conductas y monitorear los resultados de la empresa para satisfacer a los accionistas principales (Hillman \& Dalziel, 2003). Son múltiples las investigaciones que se han realizado con el fin de analizar el impacto de la junta de directores en los resultados de la compañía. La literatura pone de manifiesto cómo las actividades de responsabilidad social empresarial son cruciales en la perspectiva de la orientación al mercado, dado que la empresa debe tener la capacidad de servir a los grupos de interés, a través de su conocimiento (Brik, Rettab \& Mellahi, 2011). Realizando el análisis literario es posible indicar que es escasa la evidencia concluyente de la existencia de una relación entre las características de la mesa y el valor que esto conlleva sobre el grado de orientación de mercado de la compañía, por lo que vale la pena señalar una serie de principios teóricos que podrían confirmar una relación entre dichas variables.

En su estudio, Cabeza y Ferna (2016) analizan la relación que surge entre la responsabilidad social corporativa, la junta directiva, y los resultados de la empresa. El marco teórico de esta investigación señala que los principios detrás de las prácticas de responsabilidad social empresarial sirven para formalizar los compromisos con la sociedad y transmitir credibilidad, de tal forma que las practicas responsables con los proveedores, clientes y la comunidad tiene un efecto directo en la competitividad de la empresa, todo esto llevando a que se 
incorpore insumos especializados, se promueva la sofisticación y se genere un entorno más productivo (Porter \& Kramer, 2002). Por su parte, hace más de dos décadas Narver y Slater (1990) reconocieron la relación que existe entre la responsabilidad social y la orientación al mercado, señalando que las empresas deben ser sensibles a la expectativas y necesidades de sus clientes y competidores. Estudios más recientes confirman una relación positiva y significativa entre la existencia de un mayor número de administradores independientes en el consejo con la adopción de la responsabilidad social en los mercado emergentes (Devi et al., 2016). $\mathrm{El}$ argumento principal gira en torno a considerar que la presencia de administradores independientes aumenta la vigilancia en la gestión, lo cual reduce problemas de agencia; además, se confirma que la existencia de administradores independientes permite una alta capitalización de mercado, esto dado a la mejora de reputación que tienen los consejeros independientes como su experiencia y conocimientos.

Tomando como base la teoría de los recursos (RBV), el rendimiento corporativo de las empresas depende en gran medida de su capacidad para alinear acciones estratégicas, recursos y capacidades de una manera eficiente (Armstrong et al., 1991). En sus resultados, Cabeza y Ferna (2016) determinan la existencia de un efecto positivo de la mayor presencia de consejeros independientes sobre el valor de la empresa; esto soporta su marco teórico, que afirma que los directores independientes logran tener una relación más estrecha con las partes interesadas, conociendo mejor sus expectativas y mejorando la respuesta de la empresa hacia las demandas del mercado (Ibrahim \& Angelidis, 1995).

Otra teoría que soporta el impacto que tiene la composición del directorio en la compañía es la de la dependencia de recursos (IDT), la cual sugiere que la selección de miembros externos es una estrategia que permite hacer frente a las relaciones de la organización con su entorno, además de mejorar la reputación y credibilidad de la organización (Pfeffer \& Salancik, 1978); de esta forma, la existencia de directores externos puede mantener a la compañía orientada sobre las contingencias externas, dado que los directores independientes buscan exponer que su responsabilidad abarca más que los accionistas, siendo los mismos más conscientes de las necesidades y expectativas de los diferentes grupos que conforman la compañía, aspecto comprobado en la investigación realizada por Wang y 
Dewhirst (1992). Por lo tanto, se puede considerar que la existencia de directores independientes puede hacer que una empresa sea más sensible a los problemas externos que le afectan, propiciando mejoras y resultados superiores. Shapiro y Tang, (2015b) exponen en sus resultados evidencia de la importancia de tener miembros independiente en el consejo, dado que esto afecta positivamente el desarrollo innovador de la compañía. Su soporte valida que la presencia de actores independientes permite la obtención de activos externos, logrando representación por parte de grupos de interés importantes, lo cual hace parte de los atributos de una empresa orientada al mercado, esto si tenemos en cuenta que la mayor parte de la literatura teórica y empírica coincide en señalar que la orientación al mercado conlleva un esfuerzo innovador continuado, soportado esencialmente en hacer algo nuevo o diferente en respuesta de las condiciones del mercado (Jaworski \& Kohli, 1993; Narver \& Slater, 1990).

En el caso chileno, Lefort y Urzúa (2008) realizaron un estudio sobre la composición de los directorios en Chile y su efecto sobre el valor de las empresas, que tenía como objetivo evaluar si la presencia de directores independientes aumentaba significativamente el valor de las empresas en Chile, concluye que el aumento de la proporción de consejeros independientes tiende a incrementar el valor de la empresa en el mercado.

A partir del estudio literario realizado, proponemos la siguiente hipótesis:

H2. A mayor porcentaje de consejeros independientes, mayor es el grado de orientación al mercado.

De igual forma, se pretende verificar la posible influencia de la variable sobre las dimensiones internas de la orientación al mercado, por lo que se proponen las siguientes hipótesis complementarias.

H2a. A mayor porcentaje de consejeros independientes, mayor es el grado de orientación al cliente.

H2b. A mayor porcentaje de consejeros independientes, mayor es el grado de orientación a la competencia.

H2c. A mayor porcentaje de consejeros independientes, mayor es el grado de coordinación interfuncional.

\section{c) Estructura de la junta directiva (tamaño de la junta)}

Otro aspecto que tienen en cuanta múltiples investigaciones tiene que ver con el tamaño del Consejo de Administración. Para este caso, 
se encuentra información que resalta tanto los aspectos positivos como negativos del tamaño del directorio. El marco de investigación referente al impacto de esta variable ha tomado diferentes enfoques; algunos han confirmado su relación significativa con la mejora el desempeño de la compañía, aspectos de innovación o responsabilidad social (De Cleyn \& Braet, 2012; Mollah et al., 2012; Zahra, 2000), mientras otros han demostrado una relación negativa (Cabeza \& Ferna, 2016; Shapiro \& Tang, 2015b).

La literatura indica que las juntas pequeñas pueden facilitar la toma y el control de decisiones con el fin de mejorar la gestión (Forbes \& Milliken, 1999), mientras grandes tableros puede dificultar la toma de decisiones y caer en la difusión de la responsabilidades, lo que reduce el rendimiento de la compañía (Brown, Helland \& Smith, 2006). Este aspecto debe ser tenido en consideración con el fin de mejorar la orientación al mercado, ya que la misma se facilita cuando los altos directivos dan el énfasis necesario a dicho aspecto en cada parte de la compañía, donde se resalta la necesidad de ser sensible a la evaluación del mercado (Bernard et al., 1993). Los estudios desarrollados en entornos emergentes, como el chileno, señalan que en estos países la propiedad está altamente concentrada y por lo mismo las firmas son menos propensas a sufrir problemas de coordinaciones, pero carecen de acceso a recursos, lo que imposibilita las acciones de innovación entre otros procesos (Handoko, Smith \& Burvill, 2014). Hay autores que indican que los tableros más grandes pueden tener más ventajas, ya que pueden permitir el acceso a diferentes perspectivas y experiencias, lo cual es crucial en el desarrollo de las empresas.

En segunda medida, la literatura indica que un mayor número de directores permite a la compañía tener disponibilidad a una mayor cantidad de recursos externos (tecnológicos y financieros), que resultan ser críticos para la innovación (Adams, Hermalin \& Weisbach, 2010). Una empresa orientada al mercado, es consciente de la necesidad continua por mejorar productos, servicios y procedimientos lo que demanda utilización de recursos a nivel interno y externo, con el fin de crear lazos entre los departamentos, asegurar la calidad y satisfacción del cliente, y estar continuamente actualizados del entorno competitivo (Narver \& Slater, 1990). Por lo anterior, podríamos decir que el acceso a mayores recursos podría influir en el grado de orientación de la compañía, lo cual 
se relaciona con la existencia de un tablero de directores más grande; a través del análisis literario es posible decir que los resultados en relación a esta variable se caracterizan por diferentes resultados frente al impacto del tamaño del directorio en los resultados empresariales, siendo poco concluyente frente a los efectos de la misma. A partir de la revisión literaria y de la discusión presentada anteriormente, proponemos la siguiente hipótesis:

\section{H3. El tamaño de la junta tiene influencia sobre el grado de orientación al mercado de las compañías.}

De igual forma, se pretende verificar la posible influencia de la variable sobre las dimensiones internas de la orientación al mercado, por lo que se proponen las siguientes hipótesis complementarias.

H3a. El tamaño de la junta tiene influencia sobre el grado de orientación al cliente de las compañías. H3b. El tamaño de la junta tiene influencia sobre el grado de orientación a la competencia de las compañías.

H3c. El tamaño de la junta tiene influencia sobre el grado de coordinación interfuncional competencia de las compañías.

\section{MÉTODO DE INVESTIGACIÓN}

\subsection{Muestra}

Para el estudio realizado se consideró como muestra las 358 empresas que transan en la bolsa de valores de Santiago (Cabeza \& Ferna, 2016; Lefort \& Walker, 2005; Mollah et al., 2012; Olavarrieta \& Friedmann, 2008b; $\mathrm{S}$ et al., 1999). Estas empresas se encuentran registradas en la base de datos de la Bolsa de Comercio de Santiago, el principal mercado chileno de valores, el cual considera solo compañías que presentaron resultados de al menos tres años consecutivos (Martínez, Stöhr \& Quiroga, 2007). El uso de esta muestra permitió tener acceso a datos de fuentes secundarias de carácter confiable con el fin de consolidar las mediciones de las variables independientes.

\subsection{Mediciones e instrumento}

\subsubsection{Variables}

\section{Variable dependiente}

Para la orientación al mercado, se siguió la conceptualización propuesta por Narver y Slater (1190), que ha servido como guía en múltiples estudios (Ho \& Wu, 2010; Johnson, Dibrell \& Hansen, 2009; Riliang Qu et al., 2005; Song, et al., 2015). 
De esta forma, la medida global de la orientación al mercado se estima como el promedio de las puntuaciones medias de las tres dimensiones, de forma que se considera que estas poseen la misma importancia en el concepto de orientación al mercado. Para este caso se ha hecho uso de la escala de tres dimensiones validada por Olavarrieta et al., (1999) en su estudio realizado en Chile; dicha escala fue corregida por profesionales que garantizaron su compresión en el idioma español y fue evaluada en al ambiente de negocios chileno.

\section{Variables independientes:}

\section{Dimensiones gobierno corporativo}

La fuente de datos usados en la medición de las variables independientes procede de la información pública proporcionada por las empresas que cotizan en la bolsa a la principal entidad de supervisión en chile, la Superintendencia de Valores y Seguros (SVS). Consideramos la concentración de propiedad como la proporción de acciones propiedad del accionista controlador. (Chen, Li, Shapiro \& Zhang, 2014; Lefort \& Walker, 2005; J. Li, Lam, Qian \& Fang, 2006; Shapiro \& Tang, 2015a; Song, Wei, et al., 2015b). En relación a la estructura y composición de la junta (presencia de consejeros independientes) como variable de análisis se usó el porcentaje total de consejeros independientes considerando el tamaño total de la junta, con el objetivo de controlar la variable dados los diferentes tamaños de tableros (Cleyn \& Braet, 2012); de esta forma, se consideró el número de consejeros independientes sobre el número total de consejeros. Además, es necesario indicar que esta variable ha sido definida de la misma manera en múltiples estudios, entre ellos los de Cleyn \& Braet (2012) y Honoré, Munari, \& van Pottelsberghe de La Potterie (2015). En relación al tamaño del tablero, la misma corresponde a la representación del número total de consejeros que conforman el directorio de la compañía (Cuadrado Ballesteros, García Rubio \& Martínez Ferrero, 2014; De Cleyn \& Braet, 2012; Franken \& Cook, 2013; Honoré et al., 2015; Lin, Li \& Bu, 2015; Porter \& Kramer, 2006; Shapiro \& Tang, 2015a).

\section{Variable control (Tamaño de la empresal}

La literatura existente en el campo de gobierno corporativo y la orientación al mercado da relevancia al tamaño de la empresa como variable de control, con el fin de probar las hipótesis. Según la literatura, las empresas más grandes son capaces de realizar una mejor asignación de recursos, prestando atención de esta manera a factores 
claves de la demanda o a grupos de interés, lo que, como hemos mencionado en capítulos posteriores, hace parte de las estrategias de una empresa orientada al mercado (Lin et al., 2015; De Cleyn \& Braet, 2012). Por el contrario las pequeñas empresas carecen a menudo de financiación, experiencias y conocimiento, lo que impide la apropiaciones de estrategias de innovación (Rothwell \& Dogdson, 1994). Otros autores sostienen que las empresas pequeñas tienen mayor facilidad para modificar sus planes y son menos burocráticas, lo que favorece el reconocimiento de oportunidades y la acción eficaz frente a las mismas Day \& Nedungadi, 1994; Narver \& Slater, 1990). Dado lo anterior, el tamaño de la empresa se incluye como variable de control y se mide como el logaritmo natural de los activos totales. (Aaboen et al., 2006; Bin-sariman, Ali, Nazli \& Nor, 2016; Cuadrado Ballesteros et al., 2014; Ho \& Wu, 2010; Song, Wei, et al., 2015b; Yang \& Wang, 2014).

\section{ESTRATEGIA DE ANALISIS}

\subsection{Pretest y recolección de datos}

El cuestionario preliminar que evalúa la orientación al mercado fue sometido a una prueba piloto (pre testeo) (Ho \& Wu, 2010; D. Li et al.,
2010; Song, Wei, et al., 2015b), que se aplicó a estudiantes de las carreras de MBA en todas sus modalidades (Weekend, Sunrise, Evenning, Global) de la Facultad de Economía y Negocios de la Universidad de Chile. Posteriormente, como es recomendado en estudios previos que evalúan las variables consideras en este estudio, el cuestionario fue administrado a dueños, socios o ejecutivos principales en cargos en las empresas seleccionadas (Johnson et al., 2009, Kanagasabai, 2008; Riliang, Qu \& Ennew, 2005; Sanusi \& Pel, 2015). Este fue enviado vía mail, a través de Qualtrics, permitiendo la conveniencia de respuesta a razón de la disposición en línea vía internet de esta herramienta (Ho \& Wu, 2010; Johnson et al., 2009).

\section{ANÁLISIS DE DATOS}

\subsection{Testeo de hipótesis}

Se realiza un análisis a través de regresiones múltiples, siguiendo primordialmente el enfoque utilizado por Kohli y Jaworski, precursores en el estudio de los antecedentes de la orientación al mercado. (Mollah et al., 2012; Qu, Ennew \& Sinclair, 2005; Shapiro \& Tang, 2015b). Para realizar la primera aproximación al modelo y al estudio de las relaciones existentes entre las variables, así como la comprobación de las 
hipótesis, se realizó un análisis de correlación. Prosiguiendo con el testeo de las hipótesis, se hizo uso del análisis de regresión múltiple con el objetivo de examinar las relaciones directas entre las variables y se utilizó una medida unidimensional de la orientación al mercado como se expuso previamente en la conceptualización de las variables. Posteriormente, se estimó la siguiente ecuación.

$$
\begin{aligned}
& \text { 1. } \mathrm{Y}=\mathrm{Bo}+\mathrm{b} 1 \mathrm{X} 1+\mathrm{b} 2 \mathrm{X} 2+\mathrm{b} 3 \mathrm{X} 3+ \\
& \text { b4X4 } 4+\mathrm{E}
\end{aligned}
$$

Donde:

Y: MPOM (Orientación al mercado)

X1: CONPRO (Concentración de propiedad)

X2: INDEP (Composición directorio)

X3: BSIZE (Tamaño del directorio)

X4: FIRMS (Tamaño de la firma)

Bo:

E: (Los residuos: $\varepsilon$ )

Para el caso de las hipótesis complementarias, se tuvo en cuenta la misma ecuación expuesta, en este caso considerando cada dimensión de la escala de orientación al mercado como variable dependiente.
2. $\mathrm{Y}(\mathrm{ccl}, \mathrm{cco}, \mathrm{ci})=\mathrm{Bo}+\mathrm{b} 1 \mathrm{X} 1+$ $\mathrm{b} 2 \mathrm{X} 2+\mathrm{b} 3 \mathrm{X} 3+\mathrm{b} 4 \mathrm{X} 4+\mathrm{E}$

Donde:

Yccl: MPON(O cliente)

Ycco: MPON(O competencia)

Yci: MPON(C interfuncional)

\section{RESULTADOS}

La encuesta fue finalmente enviada a una muestra de 280 empresas. Con una fecha de corte de 12 semanas después de diferentes lapsos de envío, se recibieron un total de 120 respuesta; a partir de un proceso de revisión, se procedió a eliminar las encuestas incompletas, las empresas que no hubieran suministrado información respecto a las demás variables de estudio y la eliminación de elementos atípicos (outlier). Se registraron así un total de 101 cuestionarios, lo que resultó en una tasa efectiva de respuesta del $36 \%$. Tal tasa de respuesta es comparable con estudios previos realizados sobre la orientación al mercado en mercados emergentes (Slater y Narver, 1994; Green ley, 1995; Qu et al., 2005) (143-24.4\%), (Ho \& Wu, 2010) (127-14\%) como en estudios realizados en Chile (Olavarrieta \& Friedmann, 2008b) (116-36.6\%). 


\subsection{Fiabilidad y validez exploratoria y confirmatoria}

A partir del modelo inicial se calculó la fiabilidad para cada una de las dimensiones de orientación al mercado, a través del coeficiente Alfa de Cronbach. Las tres subescalas en las que se divide la orientación al mercado presentan coeficientes Alpha de Cronbach acordes con el límite inferior aceptado de forma general $(0,70)$. Para los tres factores se obtuvieron respectivamente $(0,91),(0,89)$ y $(0,89)$, en este caso los tres factores son evaluados como excelentes, lo que garantiza la consistencia interna de los componentes de cada factor, (George y Mallery 2003). Los factores obtenidos en el Análisis después de la Rotación Varimax, nos muestra que las cargas factoriales de las tres subdimensiones superan el 0,80 en cada uno de los ítems. Los tres factores consiguen explicar un $83,78 \%$ de la varianza de los datos originales. Con el objetivo de favorecer la validez e interpretación de los constructos, se procedió a la aplicación de un análisis confirmatorio (Long, 2015; Özer et al., 2006; Prajogo, 2015; Rapp et al., 2008); como se presenta en la figura 1 , donde se expresan las cargas estandarizadas de los parámetros y los índices de bondad y ajuste, así como el modelo que se ajusta adecuadamente a los datos. Así tenemos que el chi cuadrado de Satorra-Bentler $(\chi 2 \mathrm{~S}-\mathrm{B})$ es igual a 27,7857 , los grados de libertad son 24 , el ratio entre ambos es igual a 1,15 , y el modelo indica un buen ajuste, pues el ratio es menor de 2 . Hu y Bentler (1999) sugieren que valores de CFI y NNFI son iguales o superiores a 0,95 , e indican un buen ajuste en nuestro caso el $(\mathrm{CFI}=0.995)(\mathrm{NNFI}=0.98)$. El error cuadrático medio de aproximación (RMSEA) estima la discrepancia entre la matriz de covarianzas observada y la predicha y se sugiere un punto de corte en valores iguales o inferiores a 0,06 , para nuestro caso es de $(0,040)$ lo que denota un ajuste adecuado (tabla 1).

Las cargas factoriales de los ítems con respecto a los constructos de primer orden varían de 0,79 a 0,88 y son significativas a $p<0,01$. Por otra parte, las cargas factoriales de los factores de primer orden con respecto a los de segundo orden varían de 0,61 a 0,88 y son significativas a $p<0,01$. Como podemos notar, los valores estimados de los parámetros son todos superiores a 0,40 y estadísticamente significativos. El R2 de la relación planteada alcanza en todos los casos valores superiores a 0,40 para cada uno de los ítems, 
considerando, por tanto, que cada uno de ellos tiene facultad para predecir o explicar el grado de orientación al cliente, orientación a la competencia y coordinación interfuncional. Es así como el constructo de segundo orden explica el $88 \%$, $80 \%$ y $61 \%$ de la variación de los factores de primer orden (orienta- ción al cliente, orientación a la competencia y coordinación interfuncional, respectivamente). Por tanto, se emplea el modelo de factores de segundo orden para representar la orientación al mercado y garantizar así la realización de una medición unidimensional de la variable (figura 1).

Tabla 1. Fiabilidad y validez exploratoria y concluyente

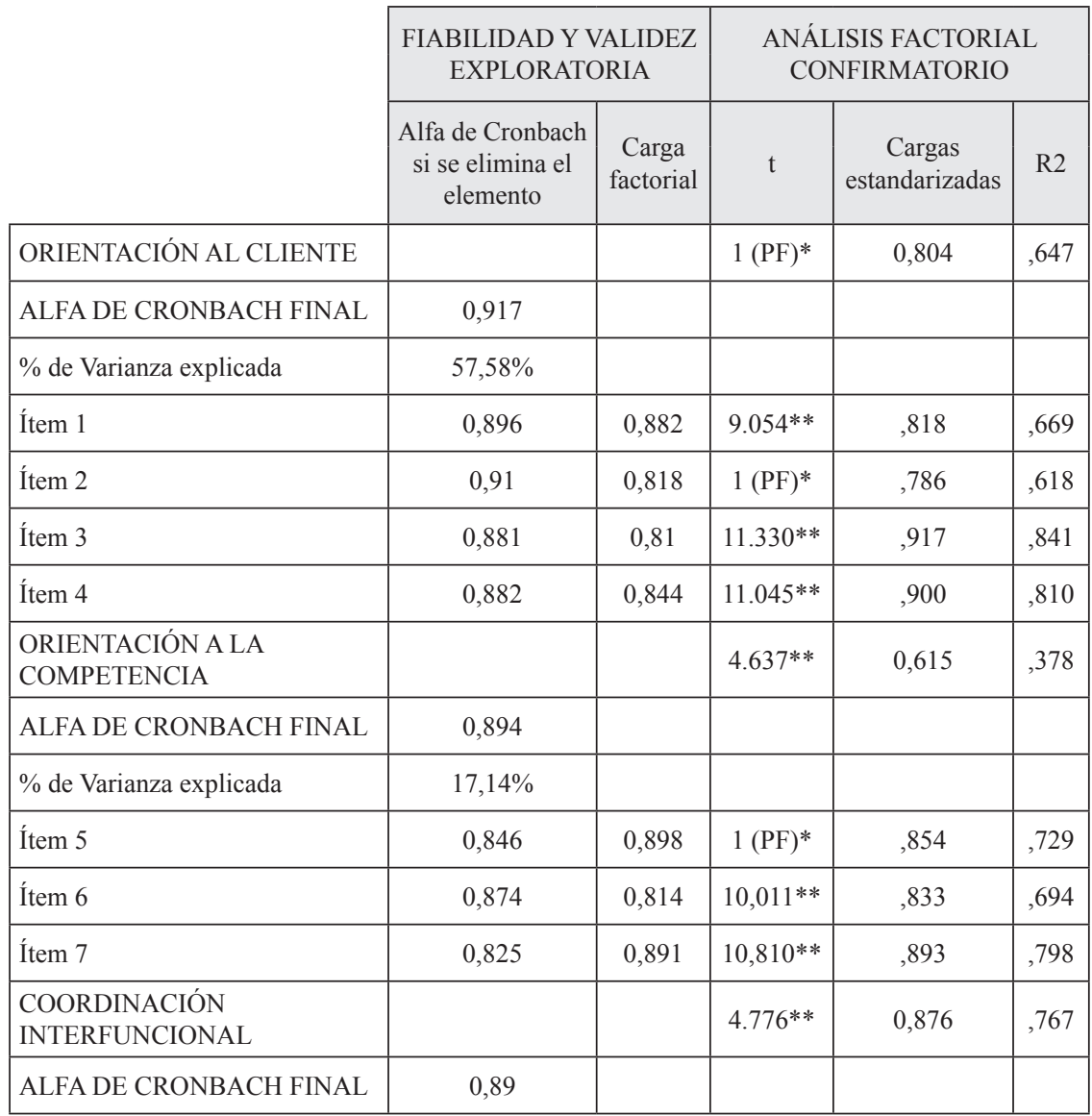




\begin{tabular}{|c|c|c|c|c|c|}
\hline & \multicolumn{2}{|c|}{$\begin{array}{l}\text { FIABILIDAD Y VALIDEZ } \\
\text { EXPLORATORIA }\end{array}$} & \multicolumn{3}{|c|}{$\begin{array}{l}\text { ANÁLISIS FACTORIAL } \\
\text { CONFIRMATORIO }\end{array}$} \\
\hline & $\begin{array}{c}\text { Alfa de Cronbach } \\
\text { si se elimina el } \\
\text { elemento }\end{array}$ & $\begin{array}{c}\text { Carga } \\
\text { factorial }\end{array}$ & $\mathrm{t}$ & $\begin{array}{c}\text { Cargas } \\
\text { estandarizadas }\end{array}$ & $\mathrm{R} 2$ \\
\hline$\%$ de Varianza explicada & $9 \%$ & & & & \\
\hline Ítem 6 & - & 0,865 & $1(\mathrm{PF})^{*}$ &, 838 & ,703 \\
\hline Ítem 7 & - & 0,849 & $9.143^{* *}$ & ,916 & 839 \\
\hline KMO & 0,84 & & & & \\
\hline E. BARTLETT & $0,00 \mathrm{sig}$ & & & & \\
\hline $\begin{array}{l}\text { Varianza explicada por los tres } \\
\text { componentes }\end{array}$ & $83,78 \%$ & & & & \\
\hline $\begin{array}{l}\text { ÍNDICES DE BONDAD Y } \\
\text { AJUSTE }\end{array}$ & & & & & \\
\hline $\begin{array}{l}\text { SATORRA-BENTLER } \\
\text { SCALED CHI-SQUARE }\end{array}$ & 1,15 & & & & \\
\hline NON-NORMED FIT INDEX & 0,985 & & & & \\
\hline COMPARATIVE FIT INDEX (CFI) & 0,990 & & & & \\
\hline
\end{tabular}

* Parámetro fijado a la unidad para fijar la escala de medida de la variable latente.

Fuente: elaboración propia.

Figura 1. Modelo de tres factores de primer orden y uno segundo (valor parámetros estandarizados)

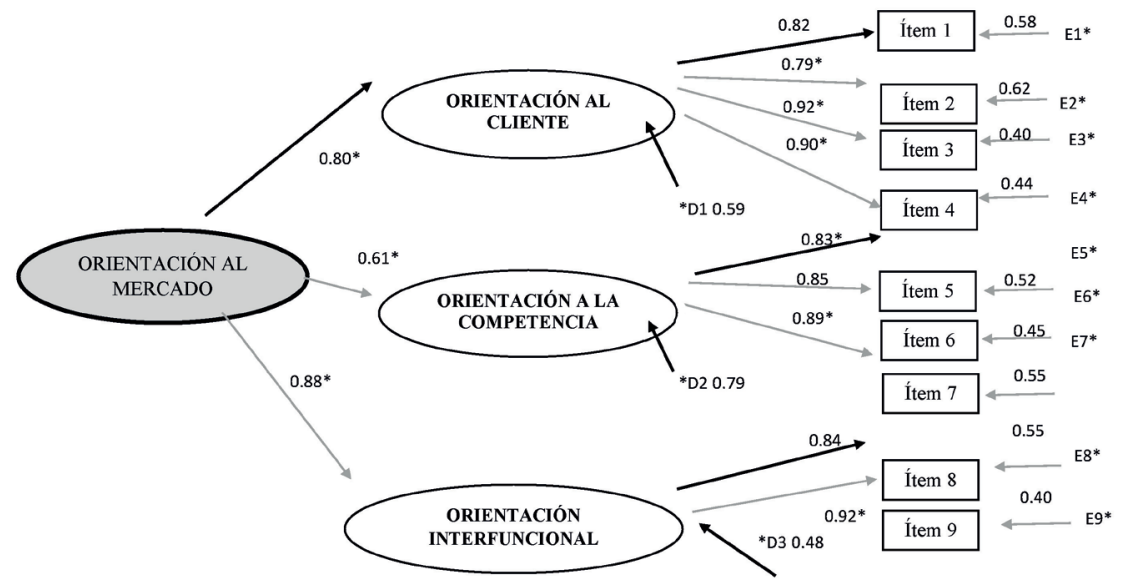

*Todos los vínculos (coeficientes) son significativos $\mathrm{p}<0,05$

Fuente: elaboración propia. 


\subsection{Testeo de hipótesis}

\subsubsection{Correlaciones}

La prueba de Kolmogorov-Smirnov arrojó que no todas las variables tienen una distribución normal para su posterior análisis mediante pruebas paramétricas. Por tanto, se procede a realizar el análisis mediante la aplicación de las pruebas no paramétricas (Bonett \& Wright, 2000). De acuerdo con lo anterior, y bajo el esquema de correlaciones no paramétricas, se procedió a aplicar dos pruebas Spearman. El análisis de correlación se llevó a cabo en todas las variables en este estudio para dos propósitos; en primer lugar, para explorar las relaciones entre las variables, y en segundo lugar, para comprobar la presencia de multicolinealidad. A partir de la información presentada en la tabla 2 es posible establecer que existe correlación entre las variables independientes, razón por la que no se pudo incorporar todas las variables con el fin de evitar problemas de multicolinealidad.

Respecto a larelación de las variables predictoras y MPON (orientación al mercado) es posible indicar que ninguna de presenta una correlación alta, confirmando que no existe una relación lineal entre las variables independientes CONPRO (concentración de propiedad $)(\mathrm{r}=0,94, \mathrm{p}>0,01)$, INDEP (\% consejeros independientes) $(\mathrm{r}=0,55, \mathrm{p}>0,01)$ y FIRMS (Tamaño de la compañía) ( $\mathrm{r}=0,31$, $\mathrm{p}>0,01)$ y la variable dependiente MPON (orientación al mercado), para esta muestra específica de empresas chilenas. Los datos muestran la existencia de una correlación baja pero significativa entre el BSIZE (tamaño de la junta directiva) y el MPON (grado de orientación al mercado) $(r=0,227, p<0,05)$, lo cual indicaría que las ventajas asociadas con la existencia de un tablero grande, como las perspectivas y la experiencia de sus miembros (Aaboen et al., 2006) y la disponibilidad a una mayor cantidad de recursos externos, (Adams et al., 2010) favorecería el uso y aplicación de estrategias enfocadas en conocer y atender adecuadamente al cliente (Cuadrado Ballesteros et al., 2014).

De igual forma, al hacer el análisis respecto a las dimensiones internas de la orientación al mercado que funcionan como variable dependiente, se muestra una relación moderada y positiva entre el grado de orientación al cliente $M P O N(O$ cliente) y BISE (tamaño del consejo de directores) $(\mathrm{r}=0,307, \mathrm{p}<0,01)$; esto pone en manifiesto la influencia que tiene el tamaño del directorio frente al acceso a recursos y la mejora puntual de la orientación 
al cliente. De igual forma, la información previamente compartida puede dar pie a la existencia de una correlación baja pero significativa entre la (coordinación interfuncional) y el BSIZE (tamaño del directorio) ( $\mathrm{r}=0,210, \mathrm{p}<0,05)$ (tabla 2); además, se ha indicado que un directorio más grande puede permitir el acceso a diferentes perspectivas y experiencia, lo que permite afirmar es necesaria la inclusión de una variedad de perspectivas sobre la estrategia corporativa, que incluya la mejora en la comunicación y el intercambio de información y recursos en las diferentes áreas (Pearce \& Zahra, 2012).

Tabla 2. Correlaciones Rho de Spearman variable dependiente dimensiones de la orientación al mercado y variables predictores

\begin{tabular}{|l|c|c|c|c|c|c|c|c|c|c|c|c|}
\hline & $\mathrm{M}$ & $\mathrm{SD}$ & $\mathrm{KS}$ & 1 & 1.1 & 1.2 & 1.3 & 2 & 3 & 4 & 5 \\
\hline MPON $^{\mathrm{a}}$ & 5,37 & 1,09 & 0,466 & 1 & & & & & & & \\
\hline $1.1 \mathrm{Ocli}^{\mathrm{b}}$ & 5,85 & 1,17 & $0,006^{*}$ &, $777^{* *}$ & 1 & & & & & & \\
\hline Ocom $^{\mathrm{c}}$ & 5,02 & 1,41 & 0,376 &, $775^{* *}$ &, $413^{* *}$ & 1 & & & & & \\
\hline Cint $^{\mathrm{d}}$ & 5,44 & 1,1 & 0,428 &, $921^{* *}$ &, $806^{* *}$ &, $872^{* *}$ & 1 & & & & \\
\hline CONPRO $^{\mathrm{e}}$ & 0,56 & 0,3 & 0,125 &,- 094 &,- 052 &,- 030 &,- 047 & 1 & & & \\
\hline INDEP $^{\mathrm{f}}$ & 0,18 & 0,19 & $0,004^{*}$ &,- 061 &,- 054 &,- 023 &,- 043 &,- 128 & 1 & & \\
\hline BSIZE $^{\mathrm{g}}$ & 7,03 & 1,66 & $0 *$ &, $227^{*}$ &, $307^{* *}$ &, 069 &, $210^{*}$ &,$- 258^{*}$ &, 155 & 1 & \\
\hline FIRMS $^{\mathrm{h}}$ & 19,42 & 2,07 & 0,661 &, 103 &, 103 &,- 034 &, 033 &, 097 &, $353^{* *}$ &, $396^{* *}$ & 1 \\
\hline
\end{tabular}

${ }^{a}$ Grado de orientación al mercado.

${ }^{\mathrm{b}}$ Orientación al cliente.

${ }^{\mathrm{C}}$ Orientación a la competencia.

${ }^{\mathrm{d}}$ Coordinación interfuncional.

e Porcentaje de acciones en propiedad del "accionista controlador".

${ }^{\mathrm{f}}$ Porcentaje consejeros independientes dentro de la junta.

${ }^{g}$ Número total de directores en la Junta.

${ }^{\mathrm{h}}$ Logaritmo natural de los activos totales.

* La correlación es significante al nivel 0,05 (bilateral).

** La correlación es significativa al nivel 0,01 (bilateral).

Fuente: elaboración propia. 


\subsubsection{Regresión}

Los resultados del análisis de regresión, se muestran en la tabla 3. Así, en primera medida, si consideramos la ecuación propuesta en este estudio (modelo 1), podemos decir que no hay poder explicativo significativo por parte de las variables independientes en el grado de orientación al mercado, pues los resultados indican que las variables concernientes al gobierno corporativo no afectan significativamente el grado de orientación de las compañías seleccionadas en esta muestra especifica de empresas. El coeficiente de correlación múltiple $\mathrm{R}=0,260$ indica la correlación entre el conjunto de variables predictoras (COMPRO, INDEP, BSIZE, FIRMS) y el criterio (MPON), $\mathrm{R} 2=6,7 \%$, por lo que el ajuste del modelo puede considerarse según este dato bastante bajo. Por otra parte, en relación con el ANOVA y su correspondiente índice $\mathrm{F}$, el modelo se muestra claramente no válido para representar los datos; el valor de significación obtenido $(\mathrm{F}=1,627 \mathrm{p}=0,174 \mathrm{p}>0,01)$ indica que es alta la probabilidad de que el conjunto de variables predictoras introducidas no sea suficiente para aportar explicación de los valores predichos de Y (MPON) (Orientación al mercado).
Sin embargo, a través del análisis de correlaciones realizado previamente sobre cada uno de los predictores, como se evidencia en la tabla 2 , nos conduce a afirmar que no todos ellos resultan igualmente relevantes. Por lo que se procedió a realizar una depuración del modelo de regresión múltiple eliminando aquellos regresores que por sus poderes predictivos "no significativos" no expliquen mucho más sobre MPON (Orientación al mercado) que lo que ya explican aquellos que sí lo son.

Mediante el análisis de correlación sabemos que la variable BSIZE (Tamaño del directorio) fue la única que resulto significativa. El modelo 3 (tabla 3 ) muestra los resultados, excluyendo las demás variables predictoras (COMPRO, INDEP), además de la variable control FIRMS que, como fue explicado anteriormente, ocasiona multicolinealidad en el modelo. La prueba Durbin Watson aproximada a $2(\mathrm{DW}=2,013)$ nos indica que se cumple correctamente el principio de que los términos de los residuos no están correlacionados entre sí; la validez del modelo mejora, donde el valor de $\mathrm{p}$ asociado al índice $\mathrm{F}$ nos indica una significación de este coeficiente, $\mathrm{p} \leq .05$, $(\mathrm{F}=5.076 \mathrm{p}=0,027 \mathrm{p}<0,05)$, por tanto podemos decir que el grado de orientación al mercado está afecta- 
do significativamente por la variable BSIZE (tamaño del directorio) $(\mathrm{t}=-$ $0,150, p=0,026 p<0,05)$. Teniendo en cuenta lo anterior, se puede decir que las empresas con un tamaño de tablero más grande están más orientadas al mercado. Los resultados apoyan de manera leve la H3, y decimos que se acepta la hipótesis nula, aceptando así la influencia de esta variable sobre el grado de orientación al mercado de la compañía, ya que la relación es positiva.

Respecto al estudio de las dimensiones, en la tabla 3 se presentan los resultados para cada uno de los modelos, considerando cada dimensión como variable independiente. El modelo 1 -donde la orientación al cliente es la variable dependiente, incluyendo todas las variables independientes consideradas en este estudio- muestra la no significancia asociada a las variables COMPRO $(p=0,875 p>0,05), \operatorname{INDEP}(p=0,334$ $p>0,05$ Y FIRMS $(p=0,931 p>0,05)$; sin embargo, a través del análisis de correlaciones realizado previamente sobre cada uno de los predictores, se llegó a la conclusión de que no todos ellos resultan igualmente relevantes, por lo que se procedió a realizar una depuración del modelo de regresión múltiple, el modelo 2 , donde la orientación al cliente es la variable dependiente; así, el valor de $\mathrm{p}$ asociado al índice $\mathrm{F}$ nos indica una significación de este coeficiente, $\mathrm{p} \leq .05,(\mathrm{~F}=9.665 \mathrm{p}=0,003 \mathrm{p}<0,05)$, por lo que el modelo resulta válido en este caso, donde además se evidencia la significancia de la variable BSIZE tamaño del directorio $(\mathrm{p}=$ $0,003 p<0,05)$.

En el modelo 3, donde la orientación a la competencia es la variable independiente, concluimos que no hay poder explicativo significativo de cualquiera de las variables predictoras sobre el grado de orientación a la competencia $(\mathrm{F}=0,212 \mathrm{p}=0,931$ $\mathrm{p}>0,05$ ). Igualmente, en los resultados de la regresión (ver tabla 3) $p$ value $>0,05$ en las variables COMPRO, INDEP Y FIRMS, da cuenta de su no significancia. 


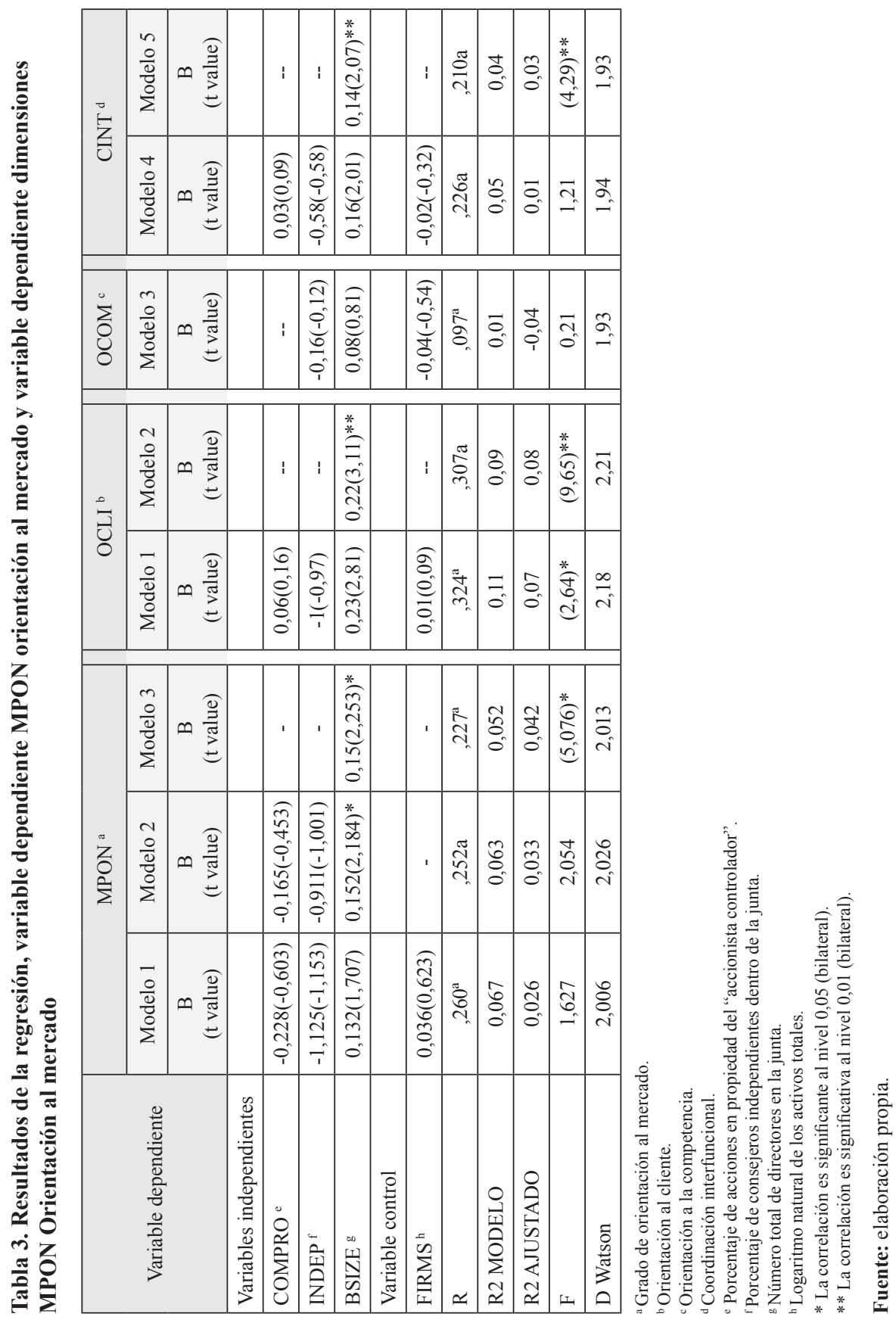


Como se evidencia en el modelo 4, donde la variable dependiente es la coordinación interfuncional, incluyendo todas las variables independientes consideradas en este estudio, el valor de significación obtenido $(F=1,210 p=0,312 p>0,05)$ (ver tabla 3) indica que es alta la probabilidad de que el conjunto de variables predictoras introducidas no sea suficiente para aportar explicación de los valores predichos de MPON coordinación interfuncional. De igual forma, el modelo muestra la no significancia asociada a las variables COMPRO ( $\mathrm{p}=0,933$ $p>0,05), \operatorname{INDEP}(p=0,561 p>0,05$ Y FIRMS ( $p=0,750 p>0,05)$, por lo que se procedió a realizar una depuración del modelo de regresión múltiple. Por su parte, el modelo 5 , donde la coordinación interfuncional es la variable dependiente, el valor de $\mathrm{p}$ asociado al índice $\mathrm{F}$ nos indica una significación de este coeficiente, $\mathrm{p} \leq .05,(\mathrm{~F}=4,268$ $p=0,041 p<0,05$ ), por lo que resulta válido en este caso, donde además se evidencia la significancia de la variable BSIZE tamaño del direc- torio $(\mathrm{p}=0,041 \mathrm{p}<0,05)$. Como podemos ver en la tabla 3 , el modelo 2 arroja resultados que dan cuenta de que el grado de orientación al cliente está afectado significativamente por la variable BSIZE (tamaño del directorio) $(t=-0,3107, p=0,03$ $\mathrm{p}<0,05$ ), dando soporte a la hipótesis H3a, la relación es positiva por lo que empresas con un tamaño de tablero más grande están más orientadas al cliente. Con el modelo 5 (tabla 3) los resultados dan cuenta de que el grado de la coordinación interfuncional está afectado significativamente por la variable BSIZE (tamaño del directorio) $(\mathrm{t}=2,071$, $p=0,041 p<0,05)$, con lo que se puede decir que aunque hay un soporte muy débil, el grado de coordinación interfuncional está afectado significativamente por la variable BSIZE (tamaño del directorio); de esta forma, los resultados de la regresión débilmente apoyan $\mathrm{H3c}$, pues la relación es positiva, por lo que empresas con un tamaño de tablero más grande cuentan con un grado mayor de coordinación interfuncional.

Tabla 4. Resumen contraste de hipótesis

\begin{tabular}{|c|c|c|}
\hline HIPÓTESIS & RESULTADOS DE REGRESIÓN & CONTRASTE DE HIPÓTESIS \\
\hline H1: & $(\mathrm{t}=-0,362, \mathrm{p}=0,718 \mathrm{p}>0,05)$ & RECHAZADA \\
\hline H1a: & $(\mathrm{t}=-0,283, \mathrm{p}=0,778 \mathrm{p}>0,05)$ & RECHAZADA \\
\hline H1b: & $(\mathrm{t}=-0,003, \mathrm{p}=0,978 \mathrm{p}>0,05)$ & RECHAZADA \\
\hline
\end{tabular}

Continúa 


\begin{tabular}{|c|c|c|}
\hline HIPÓTESIS & RESULTADOS DE REGRESIÓN & CONTRASTE DE HIPÓTESIS \\
\hline H1c: & $(\mathrm{t}=-0,067, \mathrm{p}=0,947 \mathrm{p}>0,05)$ & RECHAZADA \\
\hline & & \\
\hline $\mathrm{H} 2$ & $(\mathrm{t}=-9,968, \mathrm{p}=-0,10 \mathrm{p}>0,976)$ & RECHAZADA \\
\hline H2a. & $(\mathrm{t}=-1,038, \mathrm{p}=0,302 \mathrm{p}>0,05)$ & RECHAZADA \\
\hline H2b. & $(\mathrm{t}=-0,124, \mathrm{p}=0,901 \mathrm{p}>0,05)$ & RECHAZADA \\
\hline H2c. & $(\mathrm{t}=-0,754, \mathrm{p}=0,452 \mathrm{p}>0,05)$ & RECHAZADA \\
\hline & & \\
\hline H3: & $(\mathrm{t}=-0,150, \mathrm{p}=0,026 \mathrm{p}<0,05)$ & SE ACEPTA APOYO MODERADO \\
\hline H3a & $(\mathrm{t}=-0,3107, \mathrm{p}=0,03 \mathrm{p}<0,05)$ & SE ACEPTA APOYO MODERADO \\
\hline H3b: & $(\mathrm{t}=-0,98, \mathrm{p}=0,810 \mathrm{p}>0,05)$ & RECHAZADA \\
\hline H3c: & $(\mathrm{t}=2,071, \mathrm{p}=0,041 \mathrm{p}<0,05)$ & SE ACEPTA APOYO DÉBIL \\
\hline
\end{tabular}

Fuente: elaboración propia.

\section{CONCLUSIONES}

Las empresas están expuestas hoy en día a un entorno que implica estar consciente tanto de los diferentes escenarios económicos como de los diferentes tipos de públicos. En este orden de ideas, la orientación al mercado aparece como una estrategia fundamental de la cultura organizacional, siendo un punto de partida en el aprovechamiento de conocimientos generados a través del estudio de las las necesidades y los deseos de los clientes y del estudio de la competencia, que permita reaccionar con alternativas innovadoras en un entorno de trabajo conjunto centrado en la co-creación de valor. Esto hace que aspectos empresariales, entre ellos la cultura organización y el gobierno corpora- tivo, sean claves a la hora de desarrollar y enfocar objetivos dados los desafíos del entorno actual.

En este trabajo aportamos a la literatura, centrando la atención en la cuestión de si el gobierno corporativo afecta a los resultados del grado de orientación al mercado de las empresas en la economía emergente de Chile. Estudiamos una muestra de 101 empresas que listan en la bolsa de Santiago, en su mayoría grandes, concentradas en el sector manufacturero de servicios y de comercio al por mayor y menor. Posteriormente, se hizo uso de una medida global del grado de orientación al mercado y de medidas individuales para cada una de sus dimensiones: orientación al cliente, orientación a la competencia y 
coordinación interfuncional. Dada los escases de literatura frente al estudio de los determinantes de la orientación al mercado en Chile y a nivel Latinoamérica, los resultados de esta investigación pretenden servir como base para futuros estudios que den relevancia a la importancia de aspectos empresariales y organizacionales en la ejecución de actividades orientadas al marketing. Encontramos que el impacto de la gestión empresarial en el grado de orientación al mercado en este grupo de empresas es bajo. Encontramos poca evidencia de que las variables de gobierno corporativo tienen efectos significativos sobre la orientación al mercado. Por su parte, las regresiones mostraron que los coeficientes de las variables de gobernabilidad no son significativos, excepto el coeficiente referente al tamaño del directorio, que mostró ser marginalmente significativa y positiva. Se encontró evidencia moderada que indica que el tamaño del directorio tiene un impacto positivo sobre el grado de orientación al mercado y sobre las dimensiones internas (orientación al cliente y coordinación interfuncional); por lo tanto, el efecto marginal del grado de orientación al mercado es levemente mayor en cuanto el número de directores del tablero aumenta. De igual forma, se encontró evidencia de que el tamaño del directorio influye positivamente en el grado de orientación al cliente y de manera leve sobre la mejora en el grado de coordinación interfuncional de las compañías tenidas en cuenta en este estudio. Los hallazgos empíricos de esta investigación solo proporcionan evidencia limitada consistente con la literatura en esta área basada en países emergentes, (Lefort \& Urzúa, 2008; Song, Wang, et al., 2015). Esto podría sugerir que la relación entre el gobierno corporativo y la gestión de actividades no solo del área de orientación al mercado, sino en innovación y responsabilidad social corporativa, puede ser diferente y compleja en las económicas emergentes, (Shapiro \& Tang, 2015a; Song, Wei, et al., 2015a).

Este estudio comprueba el papel estratégico de la junta directiva, lo que pone en relieve el impacto que esteórganode dirección tiene sobreel rendimiento empresarial, complementando así la literatura existente que abala la relación positiva entre el tamaño del directorio y el rendimiento empresarial, (Fitriya \& Locke, 2012; Forbes \& Milliken, 1999; Ganguli, 2013) la implementación de la responsabilidad social corporativa (Cabeza \& Ferna, 2016) y la apropiación de estrategias orientadas a la innovación (de Cleyn \& Braet, 2012). Este estudio pretende 
animar a los investigadores de la rama del marketing que se han centrado en estudiar de manera aislada los efectos y las consecuencias de la implementación de estrategias en esta área, para dirigir una mayor atención en los determinantes y las consecuencia de las mismas, involucrando a todos los niveles y áreas organizativas que cumplen una función estratégica y corporativa de gran importancia para cualquier empresa, sea pequeña o grande.

La junta directiva, $y$ en especial el tamaño del directorio, ha sido fuente de investigación en el estudio del gobierno corporativo. En la literatura existe evidencia de la relevancia que tiene un tablero más grande en el control de problemas de agencia, esto a razón de que en este caso un mayor número de personas estarán atentas en la toma de decisiones y harán una vigilancia más eficaz. Desde la perspectiva de la dependencia de los recursos, se indica que un tablero más grande aumenta las oportunidades de generar conexiones y por lo tanto acceso a diferentes recursos; desde la teoría de la administración se indica que un tablero más grande abre las puertas a un nivel superior de conocimiento y habilidades a la hora de buscar mejoras a conflictos o implementación de estrategias (Adams et al., 2010;
Fitriya \& Locke, 2012). El mayor tamaño del tablero impacta levemente el grado de orientación al mercado; esto indicaría que la pluralidad de puntos de vista, el acceso a recursos y el mayor control respecto a las decisiones que se toman, juega un papel importante en la mejora del grado de orientación al mercado, $\mathrm{y}$ de dos de sus dimensiones en el caso chileno (orientación al cliente y coordinación interfuncional).

Los resultados de este estudio plantean un enfoque importante para la expansión de la literatura sobre la función estratégica de las juntas directivas dentro de las empresas. (Gautam \& Boeker, 2016). La literatura existente no es clara en definir hasta qué punto aspectos de estrategia empresarial afectan la ejecución y el desarrollo de actividades en el área del marketing. Como concluyen los autores Jaworski \& Kohli (1993), siempre será útil seguir explorando la relación entre la orientación al mercado y las dimensiones alternativas de rendimiento de negocio, con el objetivo de entender mejor las condiciones bajo las cuales el grado de orientación al mercado mejora, haciendo énfasis en la importancia que la misma tiene en el rendimiento de los negocios en el entorno actual. 


\section{a) Limitaciones}

Estas limitaciones pueden ser consideras con el objetivo de ampliar la investigación en esta área. En primera medida, la muestra es relativamente pequeña, lo que limita la fiabilidad de los resultados en otros contextos nacionales o en otros tipos de empresa. Es un reto para futuras investigaciones el buscar indicios de relación entre las variables que no se vieron significativas en estos resultados a través de la replicaciones. Sería valioso considerar el estudio del efecto longitudinal de las variables, pues esto ayudaría a evitar el problema de endogeneidad expuesto por algunos autores estudiosos en el área de gobierno corporativo y los efectos del directorio; esto se relaciona con que existen fuentes de heterogeneidad no observadas, por lo que no son capturados fácilmente por las variables explicativas y las variables de control del modelo propuesto, el uso de datos longitudinales permitiría confirmar la dirección de la causalidad, eliminando algunos problemas asociados a la heterogeneidad no observada.

Los resultados de esta investigación deben considerarse como provisionales dada la escasez de investigación empírica previa en esta área. Esta investigación fue diseñada para investigar los efectos de tres variables que componen el gobierno corporativo sobre la orientación al mercado. Los resultados no se deben aplicar a otros casos. En investigaciones futuras se puede dirigir la atención a estudiar de manera empírica la posibilidad de que las variables tengan efectos no lineales, dado que en este estudio no se consideró dicho aspecto que podría justificar la no significancia entre algunas variables predictoras y la orientación al mercado. La apreciación más importante de este estudio es que muchos de los argumentos existentes en la literatura no han sido confirmados por los resultados. El reto es ampliar la investigación en estas áreas y articular de igual forma el conocimiento existente con el objetivo de generar consenso sobre un mayor número de variables.

\section{REFERENCIAS}

Aaboen, L., Lindelf P., von Koch, C., \& Lfsten, H. (2006). Corporate Governance and Performance of Small High-Tech Firms in Sweden. Technovation, 26(8), 955-968.

Adams, R. B., Hermalin, B. E., \& Weisbach, M. (2010). The Role of Boards of Directors in Corporate Governance: A Conceptual Framework and Survey. Journal of Economic Literature, 48(1), 58-107. 
Armstrong, C. E., Shimizu, K., Barney, J., Eisenhardt, Kathleen M., Martin, Jeffrey A., \& Wernerfelt, B. (1991). Firm Resources and Sustained Competitive. Journal of Management, 33(6), 99-120.

Jaworski, B., \& Kohli, A. (1993). Market Orientation: Antecedents and Consequences. Journal of Marketing, 57(3): 53-70.

Bethel, J. E., \& Liebeskind, J. (1993). The Effects of Ownership Structure on Corporate Restructuring. Strategic Management Journal, 14: 15-31.

Bin-sariman, A. S., Azwadi A., Mohd N., \& Mohd N. (2016). Board of Directors' Quality and Firms' Debt Financing: The Moderating Effect of Insider Ownership - Evidence from Omani Firms. Applied Economics 48(5), 402-410.

Bonett, D. G., \& Wright, T.A. (2000). Sample Size Requirements for Estimating Pearson, Kendall and Spearman Correlations. Psychometrika, 65(1), 23-28.

Brik, A. B., Belaid R., \& Mellahi, K. (2011). Market Orientation, Corporate Social Responsibility, and Business Performance. Journal of Business Ethics, 99(3), 307-324.

Brown, W. O., Helland, E., \& Kiholm Smith, J. (2006). Corporate Philanthropic Practices. Journal of Corporate Finance, 12(5), 855-877.
Cabeza-garcia, L., Ferna, R., \& Nieto, M. (2016). Corporate Social Responsibility, Board of Directors, and Firm Performance: An Analysis of Their Relationships. Management Science, 24(10), 85-104. Casielles, R., Santos, M. L., \& Álvarez, L. I. (2001). El concepto de orientación al mercado: perspectivas, modelos y dimensiones de análisis. Documentos de trabajo, Universidad de Oviedo, Facultad de Ciencias Económicas, $\mathrm{N}^{\circ} .245,2001,29$ págs.

Chen, V. Z., Jing L., Shapiro, D., \& Xiaoxiang Z. (2014). Ownership Structure and Innovation: An Emerging Market Perspective. Asia Pacific Journal of Management, 31(1): 1-24. Recuperado de $\mathrm{http} / / /$ link.springer.com/10.1007/ s10490-013-9357-5

Cleyn, S. H., \& Braet, J. (2012). Do Board Composition and Investor. Type Influence Innovativeness in SMEs? International Entrepreneurship and Management Journal, 8(3), 285-308.

Cuadrado Ballesteros, B., García Rubio, R., \& Martínez Ferrero, J. (2014). Efecto de La Composición Del Consejo de Administración En Las Prácticas de Responsabilidad Social Corporativa. Revista de Contabilidad, 18(1), 20-31. Recuperado de http:// www.sciencedirect.com/science/ article/pii/S1138489114000193 
Dawes, J. (2000). Market Orientation and Company Profitability: Further Evidence Incorporating Longitudinal Data. Australian Journal of Management, 25(2), 173-199.

Day, G. S., \& Nedungadi, P. (1994). Managerial Representations of Competitive Advantage. The Journal of Marketing, 31-44, 58(2), 31-44. Recuperado de http://www.jstor.org/stable/ 1252267? origin $=$ crossref.

Devi, Sheela, D., Sundarasen T., \& Nakiran Rajangam, J.Y. 2016. Board Composition and Corporate Social Responsibility in an Emerging Market. Corporate Governancen, 16(1), 35-53.

Ducassy, I., \& Montandrau, S. (2015). Corporate Social Performance, Ownership Structure, and Corporate Governance in France. Research in International Business and Finance, 34, 383-396. http://linkinghub.elsevier.com/retrieve/pii/S0275531915000070.

Fitriya, F., \& Stuart L. (2012). Board Structure, Ownership Structure and Firm Performance: A Study of New Zealand Listed-Firms. Asian Academy of Management Journal of Accounting and Finance, 8(2): 43-67.

Forbes, D. P., \& Milliken, F. J. (1999). Cognition and Corporate Governance: Understanding Boards of Directors as Strategic
Decision-Making. Academy of Management Review, 24(3): 489505.

Franken, J. R. V., \& Cook, M. L. (2013). Impact of Board Structure and Process on Cooperative Performance. Recuperado de http://purl.umn.edu/149420.

Friend, I., \& Lang, L. (1988). An Empirical Test of the Impact of Self Interest on Corporate Capital Structure. The Journal of Finance, XLIII(2), 271-282.

Ganguli, S. K. (2013). Board Composition, Ownership Structure and Firm Performance: New Indian Evidence in a Unique Regulatory Environment. Journal of Chemical Information and Modeling, 53(9), 1689-99.

Hair J.F., Anderson, R.E., Tatham, R.L., \& Black, W. C. (1995). Multivariate data analysis: withreadings. NJ, USA: PrenticeHall, Inc. Upper Saddle River.

Handoko, F., Smith, A., \& Burvill, C. (2014). The Role of Government, Universities, and Businesses in Advancing Technology for SMEs' Innovativeness. Journal of Chinese Economic \& Business Studies, 12, 171-80.

Hill, Charles W. L., \& Snell S. A. (1989). Effects of Ownership Structure and Control on Corporate Productivity. Academy of Management Journal, 32(1), 25-46. 
Hillman, A. J., \& Dalziel, T. (2003). Boards of Directors and Firm Performance: Integrating Agency and Resource Dependence Perspectives. Academy of Management Review, 28(3), 383-96.

Ho S. H., Wu, J. J. B, \& Chen Y.A. (2010). Influence of Corporate Governance and Market Orientation on New Product Preannouncement: Evidence from Taiwan's Electronics Industry. Asia Pacific Management Review, 15(1), 1-14. Recuperado de http://www.scopus.com/inward/ record.url?eid $=2$ s2.0779535649 $28 \&$ partnerID $=40 \& m d 5=264 f 5$ 85bc73307e80flbc8af457ea9cf.

Honoré, F., Munari, F., \& van Pottelsberghe de La Potterie, B. (2015). Corporate Governance Practices and Companies' R\&amp;D Intensity: Evidence from European Countries. Research Policy 44(2): 533-543. Recuperado de http://linkinghub.elsevier.com/ retrieve/pii/S0048733314001917

Ibrahim, N. A., \& Angelidis, J. P. (1995). The Corporate Social Responsiveness Orientation of Board Members: Are There Differences between inside and Outside Directors? Journal of Business Ethics, 14(5), 405-410.

Badrinarayanan, I., \& Ramachandran, V. (2016). Influence of Behavioral Integration Within Top Management Teams and Board
Control on Market Orientation, Innovation, and Firm Performance. En M. Obal, N. Krey, \& C. Bushardt (Eds.), Let's Get Engaged! Crossing the Threshold of Marketing's Engagement Era. Developments in Marketing Science: Proceedings of the Academy of Marketing Science. Springer: Cham.

Jaworski, B. J., \& Kohli, A. J. (1993). Market Orientation: Antecedents and Consequences. Journal of Marketing, 57, 53-70. Johnson, A. J., Dibrell, C., \& Hansen, E. (2009). Market Orientation, Innovativeness, and Performance of Food Companies. Journal of Agribusiness, 27(1/2), 85-106. Recuperado de http://core.ac.uk/ download/pdf/6620086.pdf

Kanagasabai， K. (2008). Market Orientation and Company Performance: A Study of Selected Japanese and Sri Lankan Companies. The Journal of Faculty of Economics, Gakshuin University, 44(4), 291-308.

Klepper, S. (1996). Entry, Exit, Growth, and Innovation over the Product Life Cycle Steven Klepper. American Economic Review, 86(3), 562-83.

Kohli,A. K., \& Jaworski, B. J. (1990). Market Orientation: The Construct, Research Propositions, and Managerial Implications. Journal of Marketing, 54(2), 1-18. Recu- 
perado de http://search.proquest. com.library.capella.edu/docvie $\mathrm{w} / 227807538$ ? accountid $=27965$ \%5Cnhttp://wv9lq5ld3p.search. serialssolutions.com.library. capella.edu/?ctx_ver $=Z 39.88$ $2004 \&$ ctx enc $=$ info: ofi/ enc:UTF-8\&rfr_id=info:sid/ ProQ:abiglobal\&rft_val_ fmt=info:ofi/fmt:ke.

Lefort, F. (2008). Hacia un mejor gobierno corporativo en Chile. Abante, 11, 18-37.

Lefort, F., \& Urzúa, F. (2008). Board Independence, Firm Performance and Ownership Concentration: Evidence from Chile. Journal of Business Research, 61(6), 615-622. Recuperado de http:// linkinghub.elsevier.com/retrieve/pii/S0148296307002354.

Lefort, F., \& Walker, E. (2005). The Effect of Corporate Governance Practices on Company Market Valuation and Payout Policy in Chile. Recuperado de http:// papers.ssrn.com/sol3/papers. cfm?abstract_id $=676710$.

Lemmon, M. L., \& Lins K. V. (2003). Ownership Structure, Corporate Governance, and Firm Value: Evidence from the East Asian Financial Crisis. Journal of Finance, 58(4), 1445-1468.

Li, Dahui, Chau, P. K. Y., \& Fujun, L. (2010). Market Orientation, Ownership Type, and E-Business Assimilation: Evidence from
Chinese Firms. Decision Sciences 41(1): 115-145.

Li, J., Lam, K., Gongming, Q., \& Yongqing, F. (2006). The Effects of Institutional Ownership on Corporate Governance and Performance: An Empirical Assessment in Hong Kong. $\mathrm{Ma}$ nagement International Review, 46(3), 259-76. Recuperado de http://ezproxy.lib.vt.edu:8080/ login?url=http://search.ebscohost.com/login.aspx?direct=tru $\mathrm{e} \& \mathrm{db}=\mathrm{b}$ th\& $\mathrm{AN}=22532276 \& \mathrm{sc}$ ope $=$ site

Lin, K. Sanders, K., Sun, J, Shipton, H., \& Mooi, E. (2015). From Customer-Oriented Strategy to Organizational Financial Performance: The Role of $\mathrm{Hu}-$ man Resource Management and Customer-Linking Capability. British Journal of Management, 27, 21-37.

Lin, P. T., Bin, L., \& Danlu, B. (2015). The Relationship between Corporate Governance and Community Engagement: Evidence from the Australian Mining Companies. Resources Policy, 43, 28-39. Recuperado de http:// linkinghub.elsevier.com/retrieve/pii/S030142071400083X.

Liu, Y., Yuan, L., \& Jiaqi, X. (2011). Ownership, Strategic Orientation and Internationalization in Emerging Markets. Journal of World Business, 46(3), 381-393. 
Long, H. C. (2015). The Impact of Market Orientation and Corporate Social Responsibility on Firm Performance: Evidence from Hoang Cuu Long. Academy of Marketing Studies Journal, 19(1), 265-278.

Martínez, J. I., Stöhr, B., \& Quiroga, B. F. (2007). Family Ownership and Firm Performance: Evidence from Public Companies in Chile. Family Business Review, 20(2), 83-94.

McNaughton, R., \& Osborne, P. (2001). Market Orientation and Firm Value. Journal of Marketing, 17(5), 1-32.

De Miguel, A., Pindado, J., \& De la Torre, C. (2004). Ownership Structure and Firm Value: New Evidence from Spain. Strategic Management Journal, 25(12), 1199-1207.

Mollah, S., Al Farooque, O., \& Wares, K. (2012). "Ownership Structure, Corporate Governance and Firm Performance." Studies in Economics and Finance 29(4): 301-319. Recuperado de http:// www.emeraldinsight.com/doi/ abs/10.1108/10867371211266937

Narver, J. C., \& Slater, S. F. (1990). The Effect of a Market Orientation on Business Profitability. Journal of Marketing 54: 20-35. Olavarrieta, S., \& Friedmann, R. (2008a). Market Orientation, Knowledge-Related Resources and Firm Performance. Journal of Business Research 61(6), 623-630.

Olavarrieta S., \& Friedmann R. (2008b). Market Orientation, Knowledge-Related Resources and Firm Performance. Journal of Business Research 61(6): 623-30. http://www.sciencedirect.com/science/article/B6V7S4PYMWSN-1/2/4dfa26b5f2b91 $621 \mathrm{bfeb} 7 \mathrm{e} 0 \mathrm{~b} 526 \mathrm{db} 3 \mathrm{f} 0$.

Olavarrieta, S., Friedmann, R., \& Hidalgo, P. (1999). La Orientación Al Mercado En Una Nación En Desarrollo: Un Estudio de Validación Con Empresas Chilenas. Revista Latinoamericana de Administración, 22, 85-98.

Pearce, J.A., \& Shaker, A. Z. (2012). Board Composition from A Strategic Contingency Perspective. Journal of Management Studies, 29, 411-38.

Pfeffer, J., \& Salancik, G. R. (1978). The external control of organizations: A resource dependence perspective. New York: Harper \& Row.

Porter, M. E., \& Kramer, M. R. (2002). The Competitive Advantage of Corporate Philanthropy. Harvard business review, 80(12), 56-68,

Porter, M. E., \& Kramer, M. R. (2006). Strategy \& Society: The Link Between Competitive Advantage and Corporate Social 
Responsibility. Harvard Business Review, 84, 78-92. Recuperado de http://efbayarea.org/documents/ events/ccc2008/Mark-KramerKeynote/Strategy-Society.PDF.

Prajogo, D., \& Newman, A. (2015).

The Influence of Market Orientation on Innovation Strategies. Emerald, 11, 68-78.

Preston Lee E., \& O'Bannon, D. P. (1997). The Corporate Social-Financial Performance Relationship. A Typology and Analysis. Business \& Society, 36(4), 419-429.

Qu, R., \& Ennew, C. (2005). Developing a Market Orientation in a Transitional Economy: The Role of Government Regulation and Ownership Structure. Journal of Public Policy \& Marketing, 24(1), 82-89.

Ralston, D. A., Terpstra-Tong, J., Terpstra, R. H., Wang, X., \& Egri, C. (2006). Today's StateOwned Enterprises of China: Are They Dying Dinosaurs or Dynamic Dynamos? Strategic Management Journal, 27(9), 825-43.

Rapp, A., Schillewaert, N., \& Wei Hao, A. (2008). The Influence of Market Orientation on EBusiness Innovation and Performance: The Role of the Top Management Team. The Journal of Marketing Theory and Practice, 16(1), 7-25. Recuperado de http://www.scopus.com/inward/record.url? eid=2-s2.0$40049083105 \&$ partnerID $=$ tZOtx3y1.

Rose, J. M., Mazza, C. R., Norman, C. S., \& Rose, A. M. (2013). The Influence of Director Stock Ownership and Board Discussion Transparency on Financial Reporting Quality. Accounting, Organizations and Society, 38(5), 397-405.

Rothwell, R., \& Dogdson M. (1994). Innovation and Size of Firm. En C. Friedmman, Economics of Industrial Innovation (pp. 215-22), London: Francis Pinter.

Santos, L., Vasquez, R., \& Álvarez, L. (2002). La orientacoón al mercado como fuente de ventajas competitivas: antecedentes asociados al equipo directivo y a la estructura organizativa en las empresas industriales. Revista Asturiana de Economia, 25, 215-44.

Sanusi, H. A, \& Pel, S. (2015). "Analysis of Effects of Market Orientation , Good Corporate Governance, and Professional Leadership on Managerial Performance in Pt . Pupuk Kujang ( Persero ) Indonesia." Journal of Economics and Sustainable Development 6(10): 202-7.

Selnes, F., Jaworski, B. J., \& Kohli, A. K. (1996). Market Orientation in United States 
and Scandinavian Companies. A Cross-Cultural Study. Scandinavian Journal of Management 12(2): 139-157.

Shapiro, D., \& Tang, Y. (2015). The Effects of Corporate Governance and Ownership on the Innovation Performance of Chinese SMEs. Journal of Chinese Economic and Business Studies, 13(4), 311-335.

Shleifer, A., \& Vishny, R. W. (1997). A Survey of Corporate Governance. The Journal of Finance 52(2): 737-83.

Song, J., Wang, R., \& Tamer Cavusgil, S. (2015). State Ownership and Market Orientation in China's Public Firms: An Agency Theory Perspective. International Business Review 24(4): 690 99. http://dx.doi.org/10.1016/j. ibusrev.2014.12.003.

Song, J., Yinghong, S. W., \& Wang, R. (2015a). Market Orientation and Innovation Performance: The Moderating Roles of Firm Ownership Structures. International Journal of Research in Marketing 32(3): 319-331. Recuperado de http://linkinghub. elsevier.com/retrieve/pii/ S0167811615000415

Valenzuela, L., \& Villegas, F. (2013). Orientación al valor del cliente y el desempeño organiza- cional: propuesta de un modelo explicativo. Revista Internacional Administración \& Finanza, 6(7), 1-25.

Wang, J., \& Dudley Dewhirst, H. (1992). Boards of Directors and Stakeholder Orientation. Journal of Business Ethics 11(2): 115-23. Wang, K., \& Shailer, G. (2015). Ownership Concentration and Firm Performance in Emerging Markets: A Meta-Analysis. Journal of Economic Surveys, 29(2), 199-229.

Yang, L., \& Wang, D. (2014). The Impacts of Top Management Team Characteristics on Entrepreneurial Strategic Orientation. 52 (2), 378 - 409. Recuperado de http://www.emeraldinsight.com/ doi/abs/10.1108/MD-03-20130140 .

Yoo, C. Y., \& Jinhan, P. (2015). Corporate Charitable Contributions: Business Award Winners' Giving Behaviors. Business Ethics: A European Review., 25(1), 25-44.

Zahra, S. (2000). Entrepreneurship in Medium-Size Companies: Exploring the Effects of Ownership and Governance Systems. Journal of Management, 26(5): 947-976. Recuperado de http:// www.sciencedirect.com/science/ article/pii/S0149206300000647 (June 21, 2015). 


\section{ANEXOS}

Anexo 1: Escala de medición utilizada para medir el grado de orientación al mercado propuesta por Narver \& Slater (1990) y modificada por Olavarrieta \& Friedmann, (2008b)

Orientación al cliente:

a. Los objetivos de nuestra empresa se basan en el logro de la satisfacción de nuestros clientes.

b. Nosotros monitoreamos continuamente nuestro compromiso de servir a las necesidades de los clientes.

c. Nuestras estrategias se fundan en la convicción de que es necesario crear valor para los clientes.

d. La satisfacción de nuestros clientes es medida en forma constante y sistemática.

Orientación a la competencia:

e. Los vendedores o ejecutivos comerciales de nuestra compañía comparten información respecto a nuestros competidores.

f. Nuestra firma responde rápidamente a las acciones de nuestros competidores.

g. Los altos ejecutivos de nuestra empresa discuten regularmente las acciones de los competidores.
Coordinación interfuncional:

h. En nuestra empresa, las áreas funcionales están integradas para satisfacer las necesidades de nuestro mercado objetivo.

i. Nuestros gerentes y jefes de área saben cómo los diferentes empleados pueden contribuir a dar valor a nuestros clientes. 